JAZYKOVEDNÝ ČASOPIS, 2011, roč. 62, č. 1

\title{
SLOVAK MULTEXT-EAST MORPHOLOGY TAGSET
}

\author{
RADOVAN GARABÍK
}

L. Štúr Institute of Linguistics, Slovak Academy of Sciences, Bratislava

GARABÍK, Radovan: Slovak MULTEXT-East Morphology Tagset. Jazykovedný časopis, 2011, Vol. 62, No. 1, pp. 19 - 39. (Bratislava)

The article presents in short, concise form the The MULTEXT-East morphology tagset as specified for the Slovak language, in the form of MULTEXT-East V3 tables, following the description of other languages present in the MULTEXT-East project. The reasoning behind some of the design choices is explained; the tagset has been influenced by other MULTEXT-East languages and by the morphosyntactic tagset used in the Slovak National Corpus.

\section{Acknowledgements}

This morphosyntactic tagset has been inspired by the Czech language specification, created by Vladimír Petkevič, with the use of Slovak morphosyntactic classification developed by the Slovak National Corpus staff.

\section{Introduction}

The wide deployment of (big) language corpora is an indispensable resource for many different kinds of linguistic research and NLP (Neuro Linguistic Programming) tools development. While the ability to search in the corpus texts is valuable by itself, an intrinsic value of a corpus can be greatly increased by adding different kinds of linguistic annotation. Some of the commonly used annotations are part-of-speech (POS) tagging, lemmatisation and morphological annotation. Lemmatisation refers to finding a basic form of a (inflected) word, called lemma this is especially useful in all kinds of lexical queries, since the users do not need to concern themselves with various word forms, but concentrate on the lexemes. Part of speech tagging assigns each words in the corpus a corresponding part of speech. Lemmatisation together with POS tagging is quite powerful and sufficient level of annotation for the English language corpora, because the part of speech and lemma contain almost all the information about the word form - what little morphology English has can be almost unambiguously seen from the lemma/wordform relationship.

Working with languages with rich inflectional morphology (such as Slovak) often requires more detailed information about word's grammar categories (such as 
case, number, tense, degree, gender etc.). Morphological annotation then refers to the process and results of assigning each word such information. Two important issues arise: first, we should efficiently encode the information in a human readable form indexable by corpus processing tools (typically as a short text string), and second, we should analyse and describe the morphology/grammar categories in a sufficiently unambiguous way to make a possibility for an automatic annotation. Such an analysis usually conflates POS tagging and morphology annotation - in order to arrive at the morphological categories, we must also find out the word's POS, and it is more convenient to treat the POS as just one of the categories. The set of all the tags used to describe grammar information is called a tagset. Such analyses usually arise from traditional grammar descriptions - however, as it turns out, traditional grammars often pose serious problems for rigorous algorithmic description. The line between syntax and morphology (and sometimes semantics) is necessarily blurred, with traditional POS description often being dependent on syntactical features or even meaning of the word in question, some of the grammar categories are unnecessarily complicated and fine grained (and automatic tagging would not achieve any reasonable precision without very difficult and elaborate setup), Traditional grammars often deal only with high level literary language, omitting "less valued" style, in prescriptivism environments, some of the grammar categories are deliberately left out or frown upon, despite their common presence in the real language corpora. Therefore, arriving at the morphological tagset for a given language is a nontrivial task, and allowances must be made for features in conflict or neglecting traditional grammars. Often, some of the syntactical information will be present in such a description (e.g. dealing with POS that - usually - do not have morphology, such as particles, conjunctions, prepositions etc.), and we use the term morphosyntactic annotation for such an analysis.

At the Slovak National Corpus Department of the L'. Štúr Institute of Linguistics, the (Slovak language) texts in the corpora automatically lemmatised and morphosyntactically tagged. The tagset used is described at the department web page and is not the same as the MULTEXT-East tagset described herein. There are two other tagsets for Slovak language widely used - one has been developed at the Faculty of Mathematics and Physics, Charles University (Hajič - Vidová-Hladká, 1997) and the second one is used in the Ajka morphology analyser (Sedláček Smrž, 2001) developed at the Masaryk University in Brno. Each of these tagsets has been developed from and is functionally identical with its Czech language tagset original.

\section{About MULTEXT-East}

The EC project MULTEXT Multilingual Tools and Corpora produced linguistics resources and a freely available set of tools that are extensible, coherent and

${ }^{1}$ http://korpus.juls.savba.sk/morpho.html 
language-independent, for seven Western European languages: English, French, Spanish, Italian, German, Dutch, and Swedish (Ide - Veronis, 1994). The EC INCO-Copernicus project MULTEXT-East Multilingual Text Tools and Corpora for Central and Eastern European Languages (MTE) is a continuation of the MULTEXT project. MULTEXT-East (Dimitrova - Erjavec - Ide - Kaalep - Petkevič Tufiş, 1998) used methodologies and results of MULTEXT. MTE developed significant language resources for six Central and Eastern European (CEE) languages: Bulgarian, Czech, Estonian, Hungarian, Romanian, Slovene, as well as English.

The MTE electronic linguistics resources also include a multilingual parallel corpus (based on translations of Orwell's novel 1984) and several language-specific resources - comparable corpus, speech samples, lexica with morphosyntactic tags (covering at least the vocabulary present in the 1984 translation).

\section{About the Slovak MULTEXT-East tagset}

The Slovak language was not part of the original MULTEXT-East specification - the contents of this article describe the tagset that has been developed at the L. Štur Institute of Linguistics independently of the main MULTEXT-East project. At the time of writing this article, the MTE tagset and resources released are at the version number 3; however the release of version number 4 (that differs mostly in the internal organisation of the data and file formats, most notably converting the source files into an XML based representation) is imminent. This article describes Slovak MTE specification conforming to version 3; changes in version 4 are not very significant from a linguistic point of view.

The format of this article, especially the formatting of part of speech tables and notes intentionally closely follows the established conventions of the MULTEXTEast documentation. The article also assumes that the reader possesses knowledge about general MULTEXT-East design principles and/or knowledge about some other MULTEXT-East language tagset.

Slovak language morphology specification compatible with the MTE tagset has been developed as a projection of the Slovak morphology tagset used at the L. Štur Institute of Linguistics (Garabík, 2006), which (pragmatically) influences some parts of the specification design, especially following the need to have an automatic procedure for converting Slovak language morpfology tagset into the MTE one, which sometimes resulted in a less detailed information in the Slovak MTE tagset, especially when compared with other languages.

In this article, we provide the complete morphosyntactic specification for the MTE tagset. We do not explain the format of the tables and the terminology in details, rather we refer the reader to the MULTEXT-East documentation (MTE 2004) 


\section{Noun (N)}

\subsection{Lexicon}

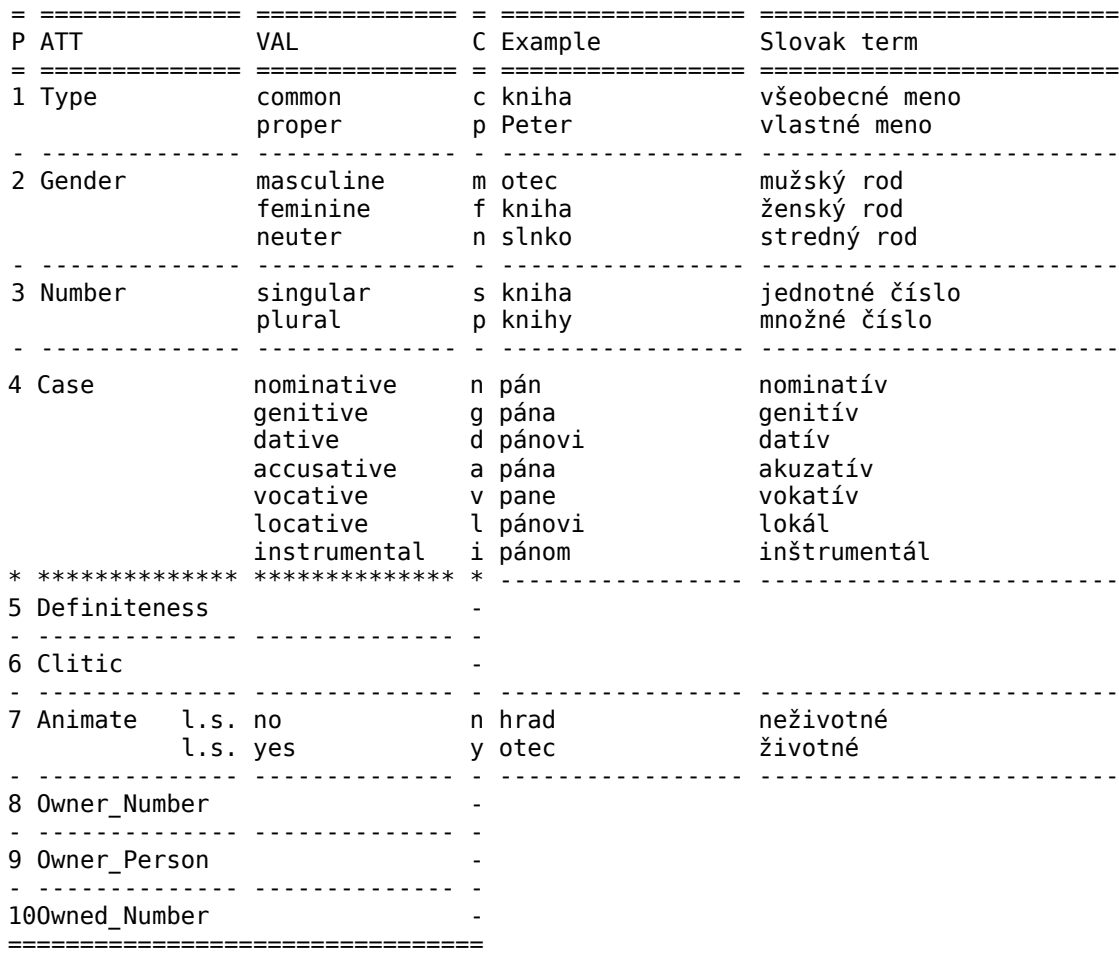

\section{Notes}

1. Slovak distinguishes masculine animate (Animate $=$ yes above) and masculine inanimate (Animate=no) Gender. Masculine inanimate nouns always have the same form in the nominative and accusative case, whereas masculine animate nouns have predominantly the same form in the genitive and accusative case. Masculine animate nouns and masculine inanimate nouns differ in accusative singular, nominative (vocative) and accusative plural only.

2. Slovak distinguishes 6 cases, the locative case being obligatorily prepositional. We fully realise there is no separate vocative case in the Slovak language morphology. What we called a "vocative" in these tables is in fact syntactical role of noun when used in addressing someone, a role that is only sometimes realised morphologically and in most of the cases is identical with the form of the nominative case. The exceptions exist in case of 
several nouns (fossilised forms of old Slavic vocative) and (considered substandard usage of) some proper names.

3. Verbal nouns are classified as nouns.

4. Adjectival nouns (gazdiná, hostinský) are classified as nouns. Sometimes the distinction between noun and adjective is not as clear as we want (typical example is obchodný cestujúci).

\subsection{Combinations}

\begin{tabular}{|c|c|c|c|c|c|c|c|}
\hline $\begin{array}{l}* * * \\
\text { PoS } \\
* * *\end{array}$ & $\begin{array}{l}* * * * \\
\text { Type } \\
* * * *\end{array}$ & $\begin{array}{l}* * * * \\
\text { Gend } \\
* * * *\end{array}$ & $\begin{array}{l}* * * * \\
\text { Numb } \\
* * * *\end{array}$ & $\begin{array}{l}* * * * \\
\text { Case } \\
* * * *\end{array}$ & $\begin{array}{l}* * * * \\
\text { Anim } \\
* * * *\end{array}$ & $\begin{array}{l}========= \\
\text { Examples } \\
=========\end{array}$ & 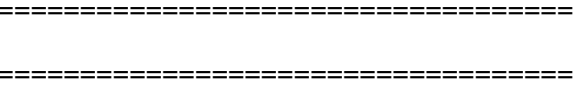 \\
\hline $\mathrm{N}$ & $\mathrm{p}$ & m & {$[\mathrm{sp}]$} & any & y & Pavol & Pavlovia \\
\hline $\mathrm{N}$ & $p$ & $\mathrm{~m}$ & {$[\mathrm{sp}]$} & any & $\mathrm{n}$ & Žiar & Žiare \\
\hline $\mathrm{N}$ & $p$ & $f$ & {$[\mathrm{sp}]$} & any & - & Lenka & Lenky \\
\hline $\mathrm{N}$ & $p$ & $\mathrm{n}$ & {$[\mathrm{sp}]$} & any & - & Branisko & Braniská \\
\hline $\mathrm{N}$ & $\mathrm{C}$ & $\mathrm{m}$ & $\mathrm{s}$ & $\mathrm{n}$ & - & chlap & dub \\
\hline $\mathrm{N}$ & C & $\mathrm{m}$ & $\mathrm{s}$ & $\mathrm{g}$ & - & chlapa & dubu/duba \\
\hline $\mathrm{N}$ & $\mathrm{C}$ & $\mathrm{m}$ & $\mathrm{s}$ & d & - & chlapovi & dubu \\
\hline $\mathrm{N}$ & C & $\mathrm{m}$ & $\mathrm{s}$ & a & $y$ & chlapa & \\
\hline $\mathrm{N}$ & C & $\mathrm{m}$ & $\mathrm{s}$ & $a$ & $\mathrm{n}$ & & dub \\
\hline $\mathrm{N}$ & C & $\mathrm{m}$ & $\mathrm{s}$ & v & - & chlape & dub \\
\hline $\mathrm{N}$ & C & $\mathrm{m}$ & $\mathrm{s}$ & $l$ & - & chlapovi & dube \\
\hline $\mathrm{N}$ & C & $\mathrm{m}$ & $\mathrm{s}$ & $i$ & - & chlapom & dubom \\
\hline $\mathrm{N}$ & C & $\mathrm{m}$ & $\mathrm{p}$ & $\mathrm{n}$ & $y$ & roboti & \\
\hline $\mathrm{N}$ & C & $\mathrm{m}$ & $\mathrm{p}$ & $\mathrm{n}$ & $\mathrm{n}$ & & roboty \\
\hline $\mathrm{N}$ & C & $\mathrm{m}$ & $\mathrm{p}$ & $g$ & - & robo & \\
\hline $\mathrm{N}$ & C & $\mathrm{m}$ & $p$ & $d$ & - & robot & \\
\hline $\mathrm{N}$ & C & $\mathrm{m}$ & $p$ & a & $y$ & robotov & \\
\hline $\mathrm{N}$ & C & $\mathrm{m}$ & $p$ & a & $n$ & & roboty \\
\hline $\mathrm{N}$ & C & $\mathrm{m}$ & $p$ & v & $y$ & roboti & \\
\hline $\mathrm{N}$ & C & $\mathrm{m}$ & $p$ & v & $n$ & & roboty \\
\hline $\mathrm{N}$ & C & $\mathrm{m}$ & $p$ & l & - & robot & och \\
\hline $\mathrm{N}$ & C & m & $p$ & i & - & robot & \\
\hline $\mathrm{N}$ & C & {$[\mathrm{fn}]$} & S & $\mathrm{n}$ & - & žena & mesto \\
\hline $\mathrm{N}$ & C & {$[\mathrm{fn}]$} & $\mathrm{S}$ & g & - & ženy & mesta \\
\hline $\mathrm{N}$ & C & {$[\mathrm{fn}]$} & $\mathrm{S}$ & d & - & žene & mestu \\
\hline $\mathrm{N}$ & C & {$[\mathrm{fn}]$} & $\mathrm{s}$ & a & - & ženu & mesto \\
\hline $\mathrm{N}$ & C & {$[\mathrm{fn}]$} & $\mathrm{s}$ & v & - & žena & mesto \\
\hline $\mathrm{N}$ & C & {$[\mathrm{fn}]$} & $\mathrm{S}$ & $l$ & - & žene & meste \\
\hline $\mathrm{N}$ & C & {$[\mathrm{fn}]$} & $\mathrm{s}$ & $i$ & - & ženou & mestom \\
\hline $\mathrm{N}$ & C & {$[\mathrm{fn}]$} & $p$ & $n$ & - & ženy & mestá \\
\hline $\mathrm{N}$ & C & [fn] & $p$ & g & - & žien & miest \\
\hline $\mathrm{N}$ & C & {$[\mathrm{fn}]$} & $p$ & $\vec{d}$ & - & ženám & mestám \\
\hline $\mathrm{N}$ & C & {$[\mathrm{fn}]$} & $p$ & $a$ & - & ženy & mestá \\
\hline $\mathrm{N}$ & C & [fn] & $p$ & v & - & ženy & mestá \\
\hline $\mathrm{N}$ & C & {$[\mathrm{fn}]$} & $p$ & $l$ & - & ženách & mestách \\
\hline $\mathrm{N}$ & $\mathrm{C}$ & {$[\mathrm{fn}]$} & $p$ & $i$ & - & ženami & mestami \\
\hline
\end{tabular}

\section{Note}

In the Combinations above, "any" is a variable standing for any admissible value. 


\section{2. $\operatorname{Verb}(V)$}

\subsection{Lexicon}

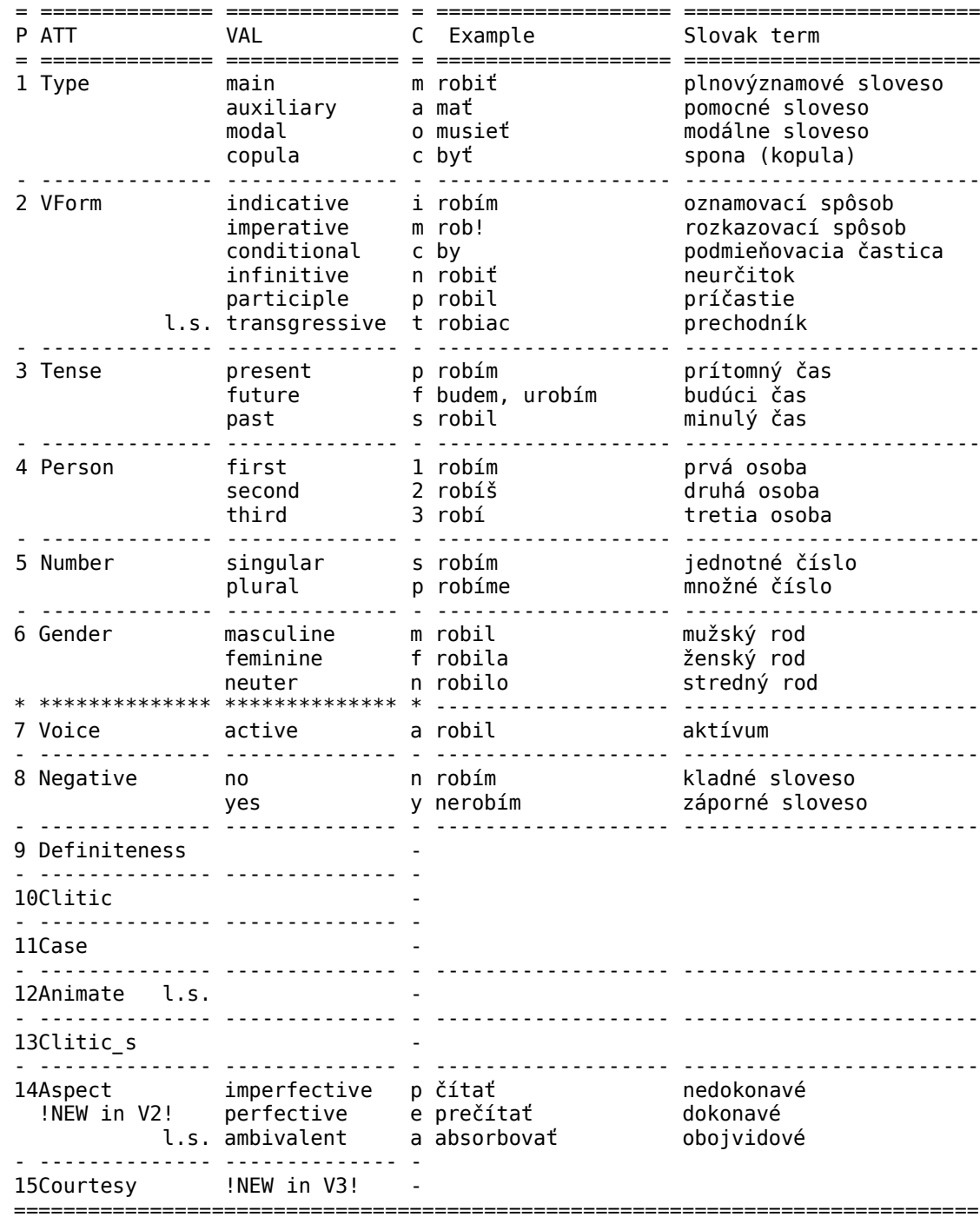

\section{Notes}

1. The verb byt' (E. "to be") in all its functions is characterized as Type $=\mathrm{c}$ (i.e. the copula), which clearly is an oversimplification because the verb has more meanings (auxiliary etc.). 
2. Auxiliary verbs (Type $=\mathrm{a})$ include neither the verb byt' (see above), nor the modal verbs, and are limited to mat', which in turn is always classified as an auxiliary verb, which is a gross oversimplification as well.

3. The "past participle" in Slovak is used for expressing compound active past Tense and is encoded as: VForm $=\mathrm{p}($ articiple), Tense $=\mathrm{s}$ (past), Voice $=\mathrm{a}$ (ctive). Note that the Czech MTE specification has also Type $=p$ (articiple) and Tense $=p($ ast $)$, here, which is clearly a mistake, because there is no Type $=p$ for verbs, and past tense has Tense $=\mathrm{s}$.

4. "Past participle" is a term taken from the common MULTEXT-East terminology, in Slovak grammars and the Slovak morphosyntactic tagset this form is called an L-participle.

5. Adjectival active and passive participles, e.g. stojaci (E. "standing") or urobený (E. "made" or "done", cf. Note 3 above) are classified as (qualificative) adjectives.

6. Negative verbs are marked as Negative $=y$, whereas non-negative verbs are marked as Negative $=n$.

7. The term "transgressive" roughly corresponds to the term "verbal participle". The transgressives have present tense and do not distinguish any other categories.

8. Gender and Animate values correspond to those associated with Nouns and are necessary to account properly for agreement.

9. Gender manifests itself in past participles only.

10. Normally, verbs form the future tense periphrastically by auxiliary byt (E. "to be") plus infinitive of the main verb. In addition to the copula, there are, however, some verbs which form future tense non-periphrastically, i.e. synthetically (Verbs of motion). Such verbal forms are marked as Tense $=\mathrm{f}$.

11. Some modal and auxiliary verbs do not form imperative and transgressive.

12. The voice value (' $a$ ' or ' $p$ ') is not specified for VForm $=c$ (onditional), VForm $=\mathrm{t}($ ransgressive) and VForm $=\mathrm{n}$ (infinitive), in which case Voice='-'.

13. Verbs form negative by prefix ne-, with the exception of the verb byt' (E. "to be") which forms the negative in indicative by using separate particle nie, e.g. nie je (is not). Here, je would be marked as positive, because of it positive form, and nie would be marked as a particle, thus apart from the presence of the particle, there is no other indication of "negativeness".

14. Ambivalent aspect can be considered a conflation of a pair of homonymous verbs, one in perfective, one in imperfective aspect. 


\subsection{Combinations}

\begin{tabular}{|c|c|c|c|c|c|c|c|c|c|c|c|}
\hline$* * *$ & $* * * *$ & $* * * *$ & $* * * *$ & $* * * *$ & $* * * *$ & $* * * * *$ & --- & --- & --- & & $====0==$ \\
\hline PoS & Type & VFrm & Tens & Pers & Numb & Gend & Voic & Neg & Anim & Asp & Examples \\
\hline$* * *$ & $* * * *$ & $* * * *$ & $* * * *$ & $* * * *$ & $* * * *$ & $* * * * *$ & --- & $-\ldots$ & $-\ldots$ & & 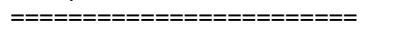 \\
\hline V & $\mathrm{m}$ & $\mathrm{n}$ & - & - & - & - & a & [ny ] & - & any & prat', neprat' \\
\hline V & a & $\mathrm{n}$ & - & - & - & - & a & {$[$ ny $]$} & - & any & mat', nemat' \\
\hline V & 0 & $\mathrm{n}$ & - & - & - & - & a & [ny ] & - & any & musiet', nemusiet' \\
\hline V & $\mathrm{C}$ & $\mathrm{n}$ & - & - & - & - & a & {$[$ ny $]$} & - & any & byt', nebyt' \\
\hline V & $\mathrm{C}$ & $\mathrm{C}$ & - & - & - & - & - & - & - & any & by \\
\hline V & C & $i$ & $f$ & [ 123] & $\mathrm{s}$ & - & a & $\mathrm{n}$ & - & any & budem, budeš, bude \\
\hline V & $\mathrm{C}$ & $i$ & $f$ & [123] & $\mathrm{s}$ & - & a & $\mathrm{y}$ & - & any & nebudem, nebudeš, nebude \\
\hline V & $\mathrm{C}$ & $i$ & $f$ & [123] & $\mathrm{p}$ & - & a & $n$ & - & any & budeme, budete, budú \\
\hline V & $\mathrm{C}$ & $i$ & $f$ & [ 123] & $p$ & - & a & $\mathrm{y}$ & - & any & nebudeme, nebudete, nebudú \\
\hline V & $\mathrm{m}$ & $i$ & $f$ & [123] & $\mathrm{S}$ & - & a & $\mathrm{n}$ & - & any & poletím, poletíš, poletí \\
\hline V & m & i & $f$ & {$[123]$} & $\mathrm{S}$ & - & a & $\mathrm{y}$ & - & $\begin{array}{l}\text { any } \\
\text { any }\end{array}$ & $\begin{array}{l}\text { nepoletím, nepoletíš, } \\
\text { nepoletí }\end{array}$ \\
\hline V & $\mathrm{m}$ & $i$ & $f$ & [ 123] & $\mathrm{p}$ & - & a & $\mathrm{n}$ & - & any & poletíme, poletíte, poletí \\
\hline V & m & i & $f$ & {$[123]$} & $\mathrm{p}$ & - & a & $\mathrm{y}$ & - & $\begin{array}{l}\text { any } \\
\text { any }\end{array}$ & $\begin{array}{l}\text { nepoletíme, nepoletíte, } \\
\text { nepoletí }\end{array}$ \\
\hline V & $\mathrm{C}$ & $i$ & $\mathrm{p}$ & [ 123] & $\mathrm{S}$ & - & a & $\mathrm{n}$ & - & any & som, si, je \\
\hline V & $\mathrm{C}$ & $i$ & $p$ & [123] & $\mathrm{S}$ & - & a & $\mathrm{y}$ & - & any & som, si, je, niet, *neni \\
\hline V & $\mathrm{C}$ & $i$ & $\mathrm{p}$ & [123] & $\mathrm{p}$ & - & a & $\mathrm{n}$ & - & any & sme, ste, sú \\
\hline V & $\mathrm{C}$ & $i$ & $\mathrm{p}$ & [ 123] & $p$ & - & a & $\mathrm{y}$ & - & any & sme, ste, sú \\
\hline V & $\mathrm{m}$ & $i$ & $p$ & [ 123] & $\mathrm{S}$ & - & a & $n$ & - & any & triem, trieš, trie \\
\hline V & $\mathrm{m}$ & $i$ & $\mathrm{p}$ & [123] & $\mathrm{S}$ & - & a & $\mathrm{y}$ & - & any & netriem, netrieš, netrie \\
\hline V & $\mathrm{m}$ & $i$ & $\mathrm{p}$ & [ 123] & $\mathrm{p}$ & - & a & $\mathrm{n}$ & - & any & trieme, triete, trú \\
\hline V & $\mathrm{m}$ & $i$ & $p$ & [123] & $p$ & - & a & $\mathrm{y}$ & - & any & netrieme, netriete, netrú \\
\hline V & a & $i$ & $\mathrm{p}$ & [ 123] & $\mathrm{S}$ & - & a & $\mathrm{n}$ & - & any & mám, máš, má \\
\hline V & a & i & $\mathrm{p}$ & {$[123]$} & $\mathrm{S}$ & - & a & $\mathrm{y}$ & - & any & nemám, nemáš, nemá \\
\hline V & a & $i$ & $\mathrm{p}$ & [ 123] & $p$ & - & a & $\mathrm{n}$ & - & any & máme, máte, majú \\
\hline V & a & $i$ & $\mathrm{p}$ & [ 123] & $p$ & - & a & $\mathrm{y}$ & - & any & nemáme, nemáte, nemajú \\
\hline V & 0 & i & $\mathrm{p}$ & [123] & $\mathrm{S}$ & - & a & $n$ & - & any & musím, musíš, musí \\
\hline V & 0 & $i$ & $\mathrm{p}$ & [ 123] & $\mathrm{S}$ & - & a & $\mathrm{y}$ & - & any & nemusím, nemusíš, nemusí \\
\hline V & 0 & $i$ & $\mathrm{p}$ & [123] & $\mathrm{p}$ & - & a & $\mathrm{n}$ & - & any & musíme, musíte, musia \\
\hline V & 0 & $i$ & $\mathrm{p}$ & [123] & $\mathrm{p}$ & - & a & $\mathrm{y}$ & - & any & nemusíme, nemusíte, nemusia \\
\hline V & $\mathrm{C}$ & $\mathrm{m}$ & $\mathrm{p}$ & 1 & $\mathrm{p}$ & - & a & [ny ] & - & any & bud'me!, nebud'me! \\
\hline V & $\mathrm{C}$ & $\mathrm{m}$ & $p$ & 2 & [sp] & - & a & $\mathrm{n}$ & - & any & bud'!, bud'te! \\
\hline V & $\mathrm{C}$ & m & $\mathrm{p}$ & 2 & {$[\mathrm{sp}]$} & - & a & y & - & any & nebud"! , nebud'te! \\
\hline V & $\mathrm{m}$ & $\mathrm{m}$ & $\mathrm{p}$ & 1 & $\mathrm{p}$ & - & a & [ny ] & - & any & pracujme!, nepracujme! \\
\hline V & $\mathrm{m}$ & $\mathrm{m}$ & $\mathrm{p}$ & 2 & [sp] & - & a & $\mathrm{n}$ & - & any & pracuj!, pracujte! \\
\hline V & $\mathrm{m}$ & $\mathrm{m}$ & $p$ & 2 & [sp] & - & a & $\mathrm{y}$ & - & any & nepracuj!, nepracujte! \\
\hline V & $\mathrm{C}$ & $\mathrm{p}$ & $\mathrm{S}$ & - & $\mathrm{S}$ & {$[\mathrm{mfn}]$} & a & $\mathrm{n}$ & - & any & bol, bola, bolo \\
\hline V & $\mathrm{C}$ & $\mathrm{p}$ & $\mathrm{S}$ & - & $\mathrm{S}$ & [mfn] & a & $\mathrm{y}$ & - & any & nebol, nebola, nebolo \\
\hline V & $\mathrm{C}$ & $p$ & $\mathrm{~S}$ & - & $\mathrm{p}$ & m & a & $\mathrm{n}$ & [yn] & any & boli \\
\hline V & $\mathrm{C}$ & $\mathrm{p}$ & $\mathrm{S}$ & - & $\mathrm{p}$ & [mfn] & a & $\mathrm{y}$ & [yn] & any & neboli \\
\hline V & a & $\mathrm{p}$ & $\mathrm{S}$ & - & $\mathrm{S}$ & {$[\mathrm{mfn}]$} & a & $\mathrm{n}$ & - & any & mal, mala, malo \\
\hline V & a & $\mathrm{p}$ & $\mathrm{S}$ & - & $\mathrm{S}$ & {$[\mathrm{mfn}]$} & a & $\mathrm{y}$ & - & any & nemal, nemala, nemalo \\
\hline V & a & $p$ & $\mathrm{~S}$ & - & $\mathrm{p}$ & {$[\mathrm{mfn}]$} & a & $\mathrm{n}$ & {$[y n]$} & any & mali \\
\hline V & a & $\mathrm{p}$ & $\mathrm{S}$ & - & $\mathrm{p}$ & {$[\mathrm{mfn}]$} & a & $\mathrm{y}$ & {$[\mathrm{yn}]$} & any & nemali \\
\hline V & 0 & $\mathrm{p}$ & $\mathrm{S}$ & - & $\mathrm{S}$ & {$[\mathrm{mfn}]$} & a & $\mathrm{n}$ & - & any & musel, musela, muselo \\
\hline V & 0 & $p$ & $\mathrm{~S}$ & - & $\mathrm{S}$ & [mfn] & a & $\mathrm{y}$ & - & any & nemusel, nemusela, nemuselo \\
\hline V & 0 & $\mathrm{p}$ & $\mathrm{S}$ & - & $\mathrm{p}$ & {$[\mathrm{mfn}]$} & a & $\mathrm{n}$ & {$[y n]$} & any & museli \\
\hline V & m & $p$ & $\mathrm{~S}$ & - & $\mathrm{S}$ & [mfn] & a & $\mathrm{n}$ & - & any & robil, robila, robilo \\
\hline V & $\mathrm{m}$ & $\mathrm{p}$ & $\mathrm{S}$ & - & $\mathrm{S}$ & [mfn] & a & $\mathrm{y}$ & - & any & nerobil, nerobila, nerobilo \\
\hline V & $\mathrm{m}$ & $\mathrm{p}$ & $\mathrm{S}$ & - & $\mathrm{p}$ & [mfn] & a & $\mathrm{n}$ & [yn ] & any & robili \\
\hline V & $\mathrm{m}$ & $p$ & $\mathrm{~S}$ & - & $p$ & [mfn] & a & $\mathrm{y}$ & [yn] & any & nerobili \\
\hline V & $\mathrm{C}$ & $\mathrm{t}$ & $\mathrm{p}$ & - & - & $-\quad-$ & a & [ny ] & - & any & súc, nesúc \\
\hline V & $\mathrm{m}$ & $\mathrm{t}$ & $\mathrm{p}$ & - & - & - & a & [ny ] & - & any & robiac \\
\hline & $* * * *$ & $k * *$ & $* * *$ & $k * *$ & $* * * *$ & $* * * * *$ & - - & & & $===$ & \\
\hline
\end{tabular}




\section{Adjective (A)}

\subsection{Lexicon}

\begin{tabular}{|c|c|c|c|c|}
\hline & $=============$ & $==============$ & $=2====$ & $====$ \\
\hline $\mathrm{P}$ & ATT & VAL & C Example & Slovak term \\
\hline & $==============$ & $==============$ & $=0$ & 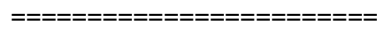 \\
\hline & & qualificative & f dobrý & vlastnostné \\
\hline & & possessive & s matkin & privlastňovacie \\
\hline & 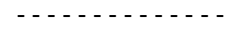 & - - - - - - - - & - - - - - - - - - - - - - - - & - - - - - - - - - - \\
\hline 2 & Degree & positive & p dobrý & 1. stupeň \\
\hline & & comparative & c lepší & 2. stupeň \\
\hline & & superlative & s najlepší & 3. stupeň \\
\hline- & $-\ldots-\ldots, \ldots$ & 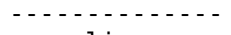 & $-\quad-\cdots--$ & 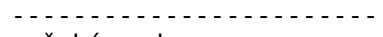 \\
\hline 3 & Gender & masculine & m dobrý & mužský rod \\
\hline & & feminine & f dobrá & ženský rod \\
\hline & & neuter & n dobré & stredný rod \\
\hline & 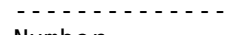 & 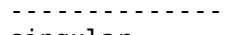 & 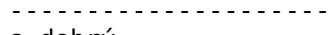 & 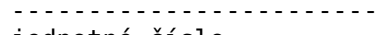 \\
\hline 4 & Number & singular & s dobrý & jednotné číslo \\
\hline & & plural & p dobrí & množné číslo \\
\hline- & $-\ldots-\ldots-\ldots$ & $-\ldots-\ldots-\ldots$ & 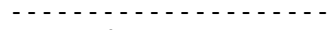 & - \\
\hline 5 & Case & nominative & n dobrý & nominatív \\
\hline & & genitive & g dobrého & genitív \\
\hline & & dative & d dobrému & datív \\
\hline & & accusative & a dobrého & akuzatív \\
\hline & & vocative & v dobrý & vokatív \\
\hline & & locative & l dobrom & lokál \\
\hline & & instrumental & i dobrým & inštrumentál \\
\hline * & $* * * * * * * * * * * * * * *$ & $k * * * * * * * * * * * * * *$ & * - - - - - & - - - - - - - - - - \\
\hline 6 & Definiteness & & - & \\
\hline & - - - - - - - - - & $-\boldsymbol{L}_{-1}$ & - & \\
\hline 7 & Clitic & & - & \\
\hline - & 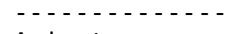 & $\ldots$ & $--\cdots--$ & 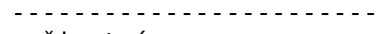 \\
\hline 8 & Animate & no & n dobré & neživotné \\
\hline & & yes & y dobrí & životné \\
\hline- & $\ldots-\ldots$ & $-\ldots-\ldots-n$ & $-\quad-\cdots-\ldots-\ldots$ & - - - - - - - - - - - - - - \\
\hline & Formation l.s. & & - & \\
\hline & 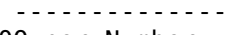 & $\ldots-\ldots$ & - & \\
\hline & OOwner_Number & & - & \\
\hline & 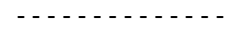 & 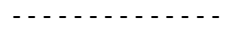 & - & \\
\hline & Lwner_Person & & - & \\
\hline & - & $\ldots \ldots$ & - & \\
\hline & OWned_Number & & - & \\
\hline
\end{tabular}

Notes

1. Two deverbative adjectival participles, i.e. past passive participle and present active participle are not distinguished. They are conflated in the ,qualificative" value of the Type attribute (Type=f). Past active participle is for all practical purposes dead in Slovak, although the form sometimes appears.

2. Only qualificative (and passive participle) Adjectives can be specified for Degree. 
3. The attributes Gender, Number, Case and Animate correspond to the same categories within the nouns. They are necessary for the proper account of agreement of adjectives with nouns.

4. Archaic short form of adjectives survives only in some words, and only in nominative (dlžen, vinen...). These are not distinguished.

5. The qualificative adjectives which have no degrees of comparison have the Degree value equal to $\mathrm{p}$ (ositive).

6. Negative adjectives have negative lemma and negativeness is not marked otherwise.

7. Adjectival nouns (gazdiná, hostinský) are classified as nouns. Sometimes the distinction between a noun and an adjective is not as clear as we want (obchodný cestujúci).

\subsection{Combinations}

\begin{tabular}{|c|c|c|c|c|c|c|c|}
\hline *** & $* * * *$ & $* * * * *$ & $* * * *$ & $* * * *$ & $* * * *$ & $* * * *$ & 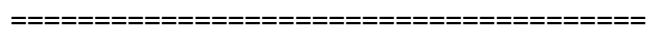 \\
\hline PoS & Type & Degr & Gend & Numb & Case & Anim & Examples \\
\hline$* * *$ & $* * * *$ & $* * * * *$ & $* * * *$ & $* * * *$ & $* * * *$ & $* * * *$ & $=0=$ \\
\hline A & $\mathrm{S}$ & - & m & $\mathrm{s}$ & $\mathrm{n}$ & - & otcov \\
\hline A & $\mathrm{S}$ & - & $\mathrm{m}$ & $\mathrm{S}$ & g & - & otcovho \\
\hline$A$ & $\mathrm{~S}$ & - & $\mathrm{m}$ & S & d & - & otcovmu \\
\hline$A$ & $\mathrm{~S}$ & - & $\mathrm{m}$ & $\mathrm{s}$ & a & $\mathrm{y}$ & otcovho \\
\hline A & $\mathrm{S}$ & - & $\mathrm{m}$ & $\mathrm{s}$ & a & $n$ & otcov \\
\hline$A$ & $\mathrm{~s}$ & - & $\mathrm{m}$ & $\mathrm{s}$ & v & - & otcov! \\
\hline$A$ & $\mathrm{~s}$ & - & $\mathrm{m}$ & s & l & - & otcovom \\
\hline A & $\mathrm{s}$ & - & $\mathrm{m}$ & s & i & - & otcovým \\
\hline$A$ & $\mathrm{~S}$ & - & $\mathrm{m}$ & $\mathrm{p}$ & $\mathrm{n}$ & $\mathrm{y}$ & otcovi \\
\hline A & $\mathrm{S}$ & - & $\mathrm{m}$ & $p$ & $\mathrm{n}$ & $n$ & otcove \\
\hline$A$ & $\mathrm{~s}$ & - & $\mathrm{m}$ & $p$ & g & - & otcových \\
\hline$A$ & $\mathrm{~S}$ & - & $\mathrm{m}$ & $\mathrm{p}$ & d & - & otcovým \\
\hline$A$ & $\mathrm{~S}$ & - & $\mathrm{m}$ & $p$ & a & $\mathrm{y}$ & otcových \\
\hline$A$ & $\mathrm{~S}$ & - & $\mathrm{m}$ & $\mathrm{p}$ & a & $\mathrm{n}$ & otcove \\
\hline A & $\mathrm{s}$ & - & $\mathrm{m}$ & $\mathrm{p}$ & v & $\mathrm{y}$ & otcovi! \\
\hline$A$ & $\mathrm{~s}$ & - & $\mathrm{m}$ & $p$ & v & $\mathrm{n}$ & otcove! \\
\hline$A$ & $\mathrm{~s}$ & - & $\mathrm{m}$ & $p$ & l & - & otcových \\
\hline$A$ & $\mathrm{~S}$ & - & m & $p$ & i & - & otcovými \\
\hline$A$ & $\mathrm{~S}$ & - & {$[\mathrm{fn}]$} & S & $\mathrm{n}$ & - & otcova, otcovo/otcove \\
\hline$A$ & $\mathrm{~S}$ & - & {$[\mathrm{fn}]$} & $\mathrm{s}$ & g & - & otcovej, otcovho \\
\hline$A$ & $\mathrm{~S}$ & - & {$[\mathrm{fn}]$} & $\mathrm{s}$ & d & - & otcovej, otcovmu \\
\hline$A$ & $\mathrm{~s}$ & - & {$[\mathrm{fn}]$} & S & a & - & otcovu, otcovo/otcove \\
\hline$A$ & $\mathrm{~S}$ & - & {$[\mathrm{fn}]$} & $\mathrm{s}$ & v & - & otcova, otcovo/otcove \\
\hline A & $\mathrm{S}$ & - & {$[\mathrm{fn}]$} & $\mathrm{s}$ & l & - & otcovej, otcovom \\
\hline$A$ & $\mathrm{~S}$ & - & {$[\mathrm{fn}]$} & $\mathrm{s}$ & $i$ & - & otcovou, otcovým \\
\hline$A$ & $\mathrm{~S}$ & - & {$[\mathrm{fn}]$} & p & $\mathrm{n}$ & - & otcove \\
\hline$A$ & $\mathrm{~s}$ & - & {$[\mathrm{fn}]$} & $p$ & g & - & otcových \\
\hline$A$ & $\mathrm{~S}$ & - & {$[\mathrm{fn}]$} & $\mathrm{p}$ & d & - & otcovým \\
\hline$A$ & $\mathrm{~s}$ & - & {$[\mathrm{fn}]$} & $p$ & a & - & otcove \\
\hline$A$ & $\mathrm{~s}$ & - & {$[\mathrm{fn}]$} & $p$ & v & - & otcove! \\
\hline$A$ & $\mathrm{~s}$ & - & {$[\mathrm{fn}]$} & $p$ & l & - & otcových \\
\hline
\end{tabular}




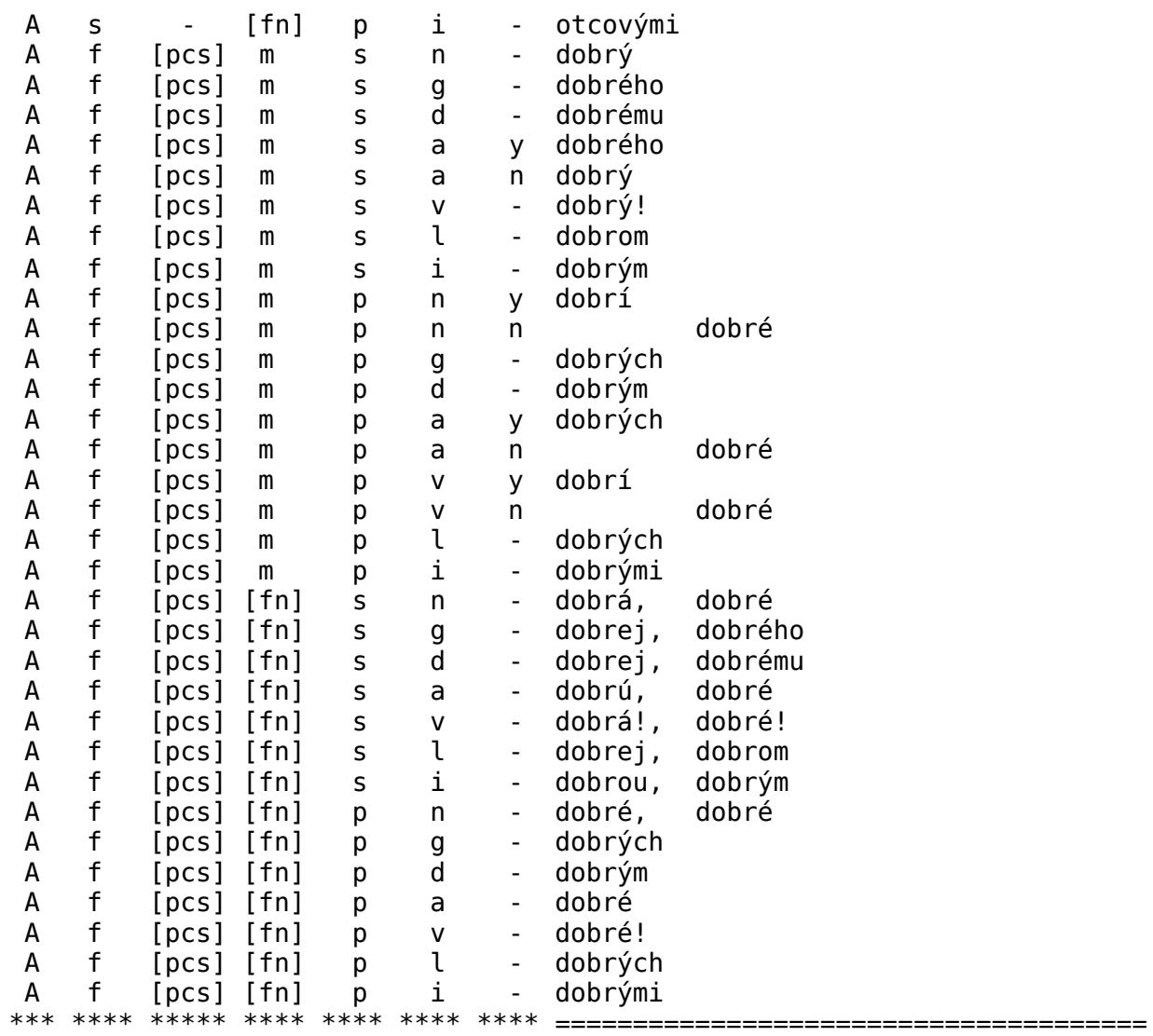

\section{Pronoun (P)}

\subsection{Lexicon}

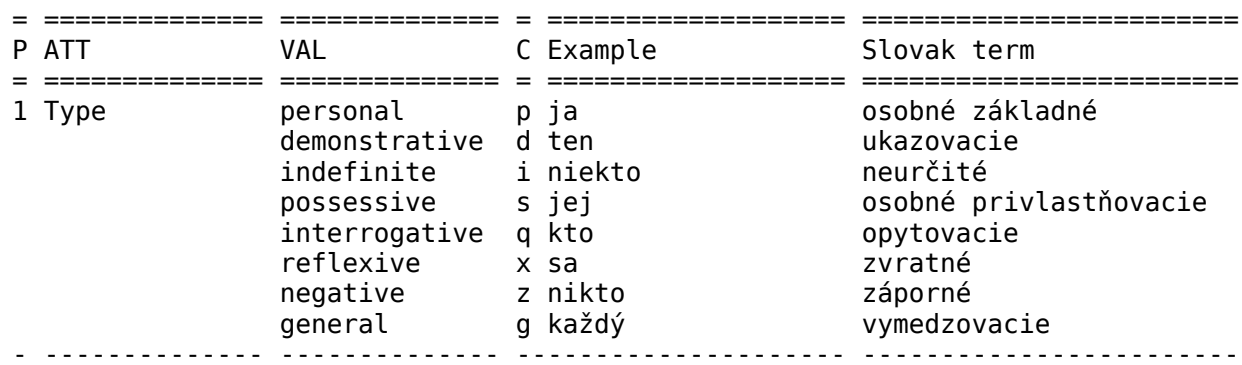




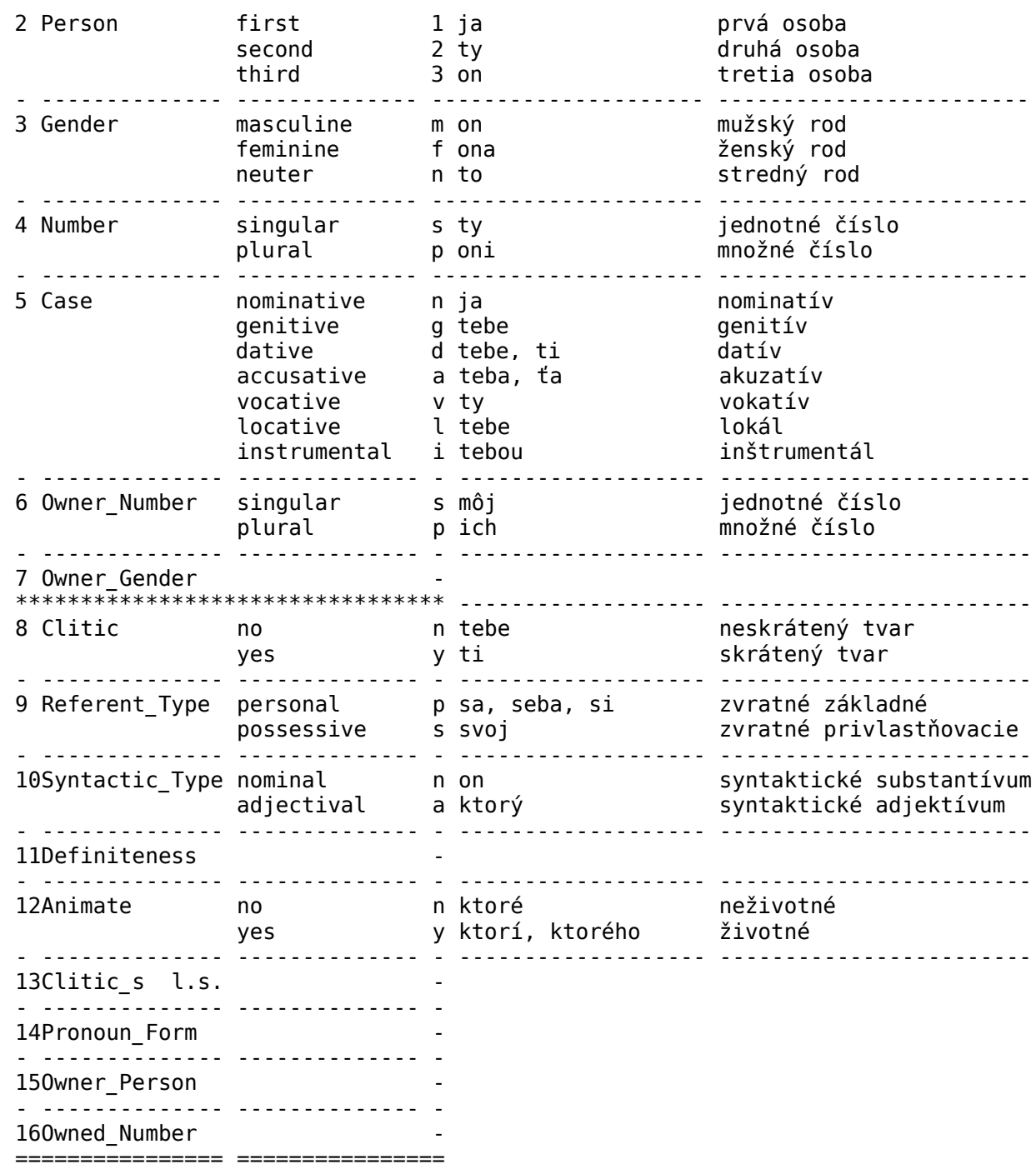

\section{Notes}

1. Gender, Number, Case and Animate correspond to the same categories as specified for nouns. They are necessary for the proper account of agreement of adjectival pronouns with Nouns.

2. Type=reflexive encompasses all reflexive pronouns (sa, sebe, si, svoj, seba) as well as $s a$ in its role as the obligatory particle of reflexive verbs. Personal and possessive reflexives are further distinguished via the Referent 
Type attribute. $S a$ in all its roles will be marked as the reflexive personal clitic pronoun.

3. Pronouns are distinguished between having a (syntactically) nominal and (syntactically) adjectival function. All pronominal types except the demonstrative and possessive one can be nominal, and all except for the personal one can be adjectival.

4. Referent_Type is used to distinguish personal reflexives (which include $s a$ in all its functions) from the possesive reflexives ( $(s v o j)$.

5. Negative and general pronouns ("general" Pronouns concern the Pronouns like $v \check{s} e t c i, k a z ̌ d y ́$ etc.) are important from the viewpoint of their syntactic distribution.

6. The Clitic attribute distinguishes clitical vs. nonclitical pronominal forms, e.g. $t i$ vs. tebe.

7. Owner_Number concern the possessor's number.

8. Owner_Gender is not marked.

9. ty ("you") is usually marked as vocative (cf. description of vocative for the Noun category). Many other pronouns can be marked as vocative because of their syntactical position, e.g. in môj bože ("“my god"), môj is marked as vocative.

10. ten, tá, to, on, ona, ono ( $3^{\mathrm{rd}}$ person demostrative and personal pronouns) have separate lemmas for each gender.

\subsection{Combinations}

\begin{tabular}{|c|c|c|c|c|c|c|c|c|c|c|c|c|}
\hline $\begin{array}{l}\text { PoS } \\
* * *\end{array}$ & $\begin{array}{l}\text { Type } \\
* * * *\end{array}$ & $\begin{array}{l}\text { Pers } \\
* * * * *\end{array}$ & $\begin{array}{l}\text { Gend } \\
* * * * *\end{array}$ & $\begin{array}{l}\text { Numb } \\
* * * *\end{array}$ & $\begin{array}{l}\text { Case } \\
* * * *\end{array}$ & $\begin{array}{l}0 \mathrm{~W} N \mathrm{~N} \\
* * \bar{*} *\end{array}$ & $\begin{array}{l}\text { OW G } \\
* * \bar{*} *\end{array}$ & Clit & $\begin{array}{l}\text { Ref } \\
---\end{array}$ & Syn & Anim & $\begin{array}{l}\text { Examples } \\
===========\end{array}$ \\
\hline $\mathrm{P}$ & $\mathrm{x}$ & - & - & - & $a$ & - & - & $y$ & $\mathrm{p}$ & $\mathrm{n}$ & - & sa \\
\hline$P$ & $x$ & - & - & - & [ga] & - & - & $\mathrm{n}$ & $\mathrm{p}$ & $\mathrm{n}$ & & seba \\
\hline$P$ & $x$ & - & - & - & d & - & - & $y$ & $p$ & $\mathrm{n}$ & - & $\mathrm{si}$ \\
\hline$P$ & $x$ & - & - & - & {$[\mathrm{dl}]$} & - & - & $n$ & $p$ & $n$ & - & sebe \\
\hline$P$ & $x$ & - & - & - & $i$ & - & - & $n$ & $\mathrm{p}$ & $n$ & - & sebou \\
\hline$P$ & $x$ & - & {$[\mathrm{mfn}]$} & all & any & - & - & $\mathrm{n}$ & S & a & [ny- ] & svoj \\
\hline$P$ & $\mathrm{p}$ & 1 & - & [sp] & all & - & - & [ny ] & - & $\mathrm{n}$ & - & ja, my \\
\hline$P$ & $\mathrm{p}$ & 2 & - & [sp] & all & - & - & [ny] & - & $\mathrm{n}$ & - & ty, vy \\
\hline$P$ & $p$ & 3 & {$[\mathrm{mfn}]$} & [sp] & all & - & - & [ny ] & - & $n$ & - & on, oni, ony, ona \\
\hline$P$ & $\mathrm{~s}$ & 1 & {$[\mathrm{mfn}]$} & all & all & [sp] & - & $n$ & - & a & [ny- ] & môj, náš \\
\hline$P$ & $\mathrm{~s}$ & 2 & {$[\mathrm{mfn}]$} & all & all & [sp] & - & $\mathrm{n}$ & - & a & [ny- ] & tvoj, váš \\
\hline$P$ & s & 3 & [mfn] & all & all & [sp] & - & $\mathrm{n}$ & - & a & [ny- ] & jeho, ich \\
\hline$P$ & s & 3 & {$[\mathrm{mfn}]$} & all & all & [sp] & - & $\mathrm{n}$ & - & a & - & jej, ich \\
\hline$P$ & d & - & {$[\mathrm{mfn}]$} & all & all & - & - & $n$ & - & a & [ny- ] & 1) \\
\hline$P$ & $i$ & - & {$[\mathrm{mn}]$} & $\mathrm{s}$ & all & - & - & $\mathrm{n}$ & - & $\mathrm{n}$ & - & 2) \\
\hline$P$ & $i$ & - & {$[\mathrm{mfn}]$} & [sp] & all & - & - & $n$ & - & a & [ny- ] & 3) \\
\hline$P$ & $q$ & - & [mn ] & $\mathrm{s}$ & all & - & - & $\mathrm{n}$ & - & $n$ & - & 4) \\
\hline$P$ & q & - & [mfn] & [sp] & all & - & - & $n$ & - & a & [ny - ] & 5) \\
\hline $\mathrm{P}$ & z & - & [mn ] & $\mathrm{s}$ & all & - & - & $\mathrm{n}$ & - & $\mathrm{n}$ & - & 6) \\
\hline$P$ & z & - & [mfn] & [sp] & all & - & - & $n$ & - & a & [ny-] & 7) \\
\hline$P$ & $\mathrm{~g}$ & - & [mfn] & [sp] & all & - & - & $n$ & - & $n$ & [ny-] & 8) \\
\hline$* * *$ & $* * * *$ & $* * * * *$ & $* * * * *$ & $* * * *$ & $* * * *$ & $* * * *$ & $* * * *$ & $\cdots$ & $\ldots$ & $-\cdots$ & $\ldots \ldots$ & $===========$ \\
\hline
\end{tabular}


1) ten, tento, tamten, taký, onaký

2) niekto, nejaký, bohviekto, hocikto, hocičo, niečo

3) ktovieaký, hocijaký

4) kto, čo

5) aký, čí

6) nikto, nič

7) žiadny, nijaký

8) každý

\section{Determiner (D)}

Not applicable.

\section{Article (T)}

Not applicable.

\section{Adverb (R)}

\subsection{Lexicon}

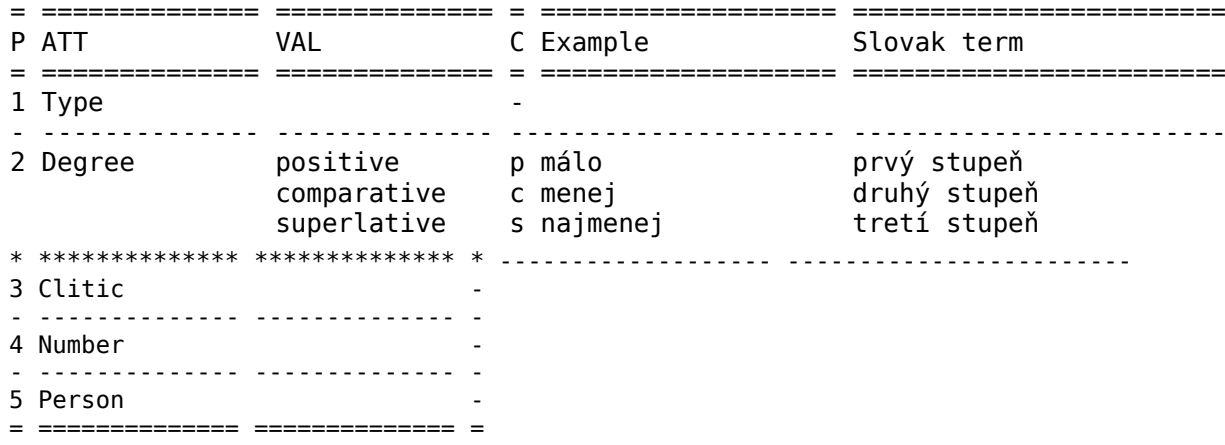

\section{Note}

1. Particles form a separate part of speech category (see below) as is customary in Slovak grammars.

2. The adverbs which have no degrees of comparison have the Degree value equal to $\mathrm{p}$ (ositive) similarly to adjectives.

\subsection{Combinations}

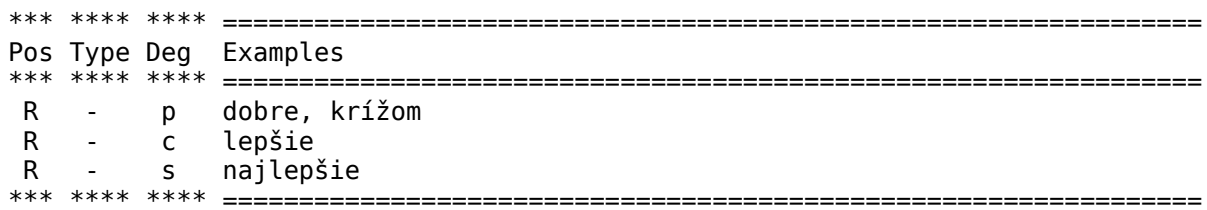




\section{Adposition (S)}

\subsection{Lexicon}

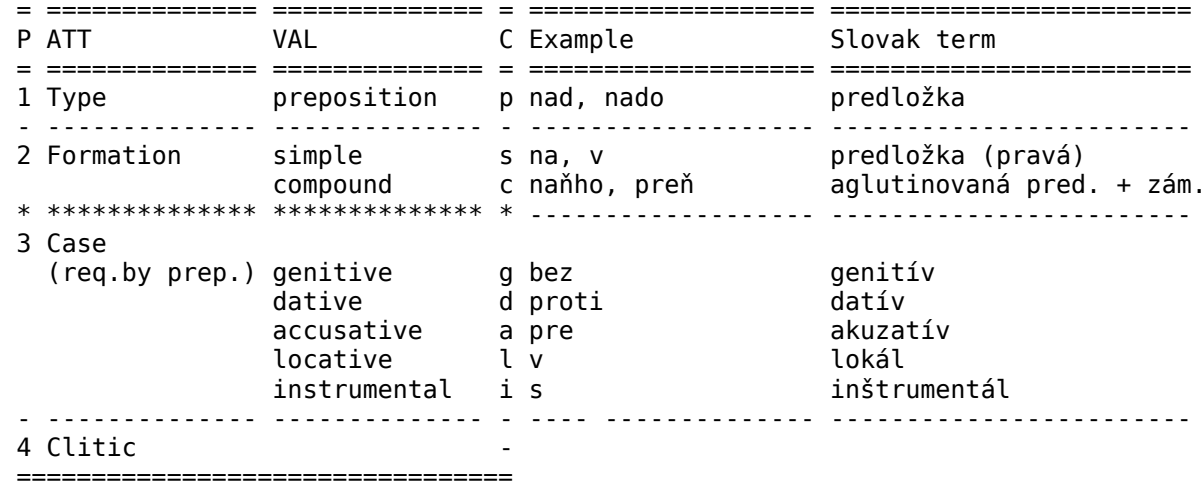

\section{Notes}

1. Slovak has only prepositions, no postpositions.

2. For the disambiguation of word forms belonging to declension parts of speech it seems necessary to include the information about the case which each preposition requires.

3. A preposition can be contracted with a pronoun; such a preposition has Formation $=\mathrm{c}$ (ompound).

4. Prepositions can be vocalized $(v \rightarrow v o)$ if following word starts with certain consonant class. These are not specially marked.

5. Compound prepositions (preposition+pronoun) do not have the case category. Arguably, this kind of words can be assigned a case rather unambiguously, more ambiguously we can classify them as pronouns. In traditional grammar, they are analysed as two separate words, i. e. two different parts of speech (preposition followed by a pronoun). We decided to keep it in the preposition category and without a case, to be consistent with other MTE typology.

6. Loanword $\grave{a}$ (often mistakenly written as $a$ ) is not considered to be a preposition (that binds with the nominative), but a particle instead.

\subsection{Combinations}

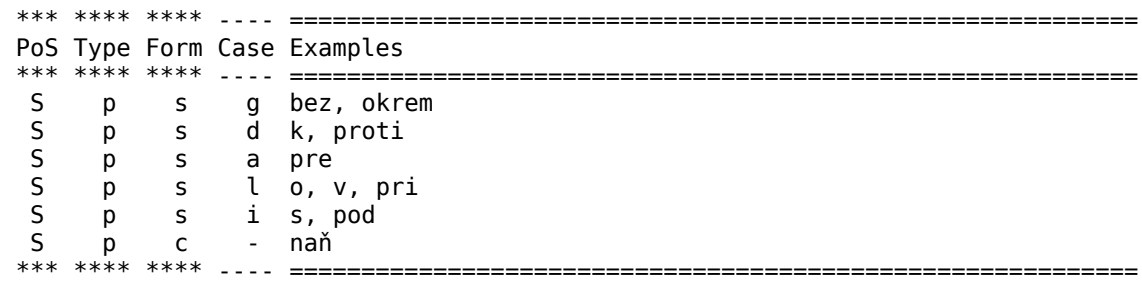




\section{Conjunction (C)}

\subsection{Lexicon}

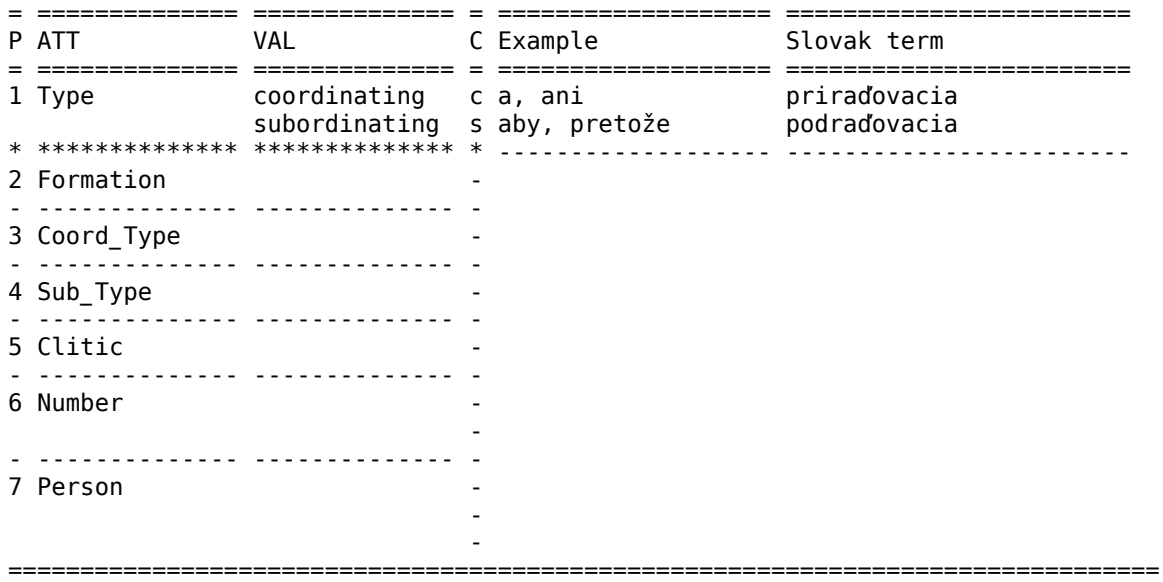

\section{Note}

The class of two-part Conjunctions has not been introduced, thus we ignore the Formation attribute.

\subsection{Combinations}

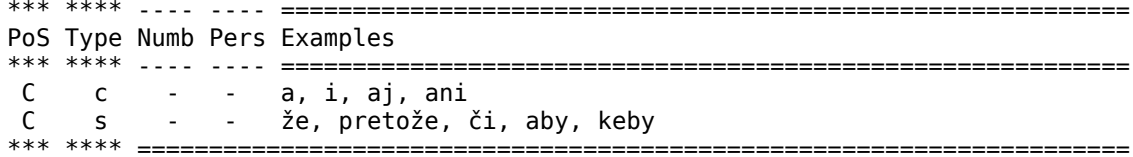

\section{Numeral (M)}

\subsection{Lexicon}

\begin{tabular}{|c|c|c|c|}
\hline ATT & VAL & C Example & Slovak term \\
\hline$===========$ & $============$ & 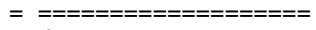 & ="==" \\
\hline Type & $\begin{array}{l}\text { cardinal } \\
\text { ordinal } \\
\text { multiple } \\
\text { special }\end{array}$ & $\begin{array}{l}\text { c dva } \\
\text { o piaty } \\
\text { m dvakrát } \\
\text { s dvojaký }\end{array}$ & $\begin{array}{l}\text { číslovka základná } \\
\text { číslovka radová } \\
\text { číslovka násobná } \\
\text { číslovka druhová }\end{array}$ \\
\hline Gender & $\begin{array}{l}\text { masculine } \\
\text { feminine } \\
\text { neuter }\end{array}$ & $\begin{array}{l}\text { m dva } \\
\text { f dve } \\
\text { n dve }\end{array}$ & $\begin{array}{l}\text { mužský rod } \\
\text { ženský rod } \\
\text { stredný rod }\end{array}$ \\
\hline
\end{tabular}




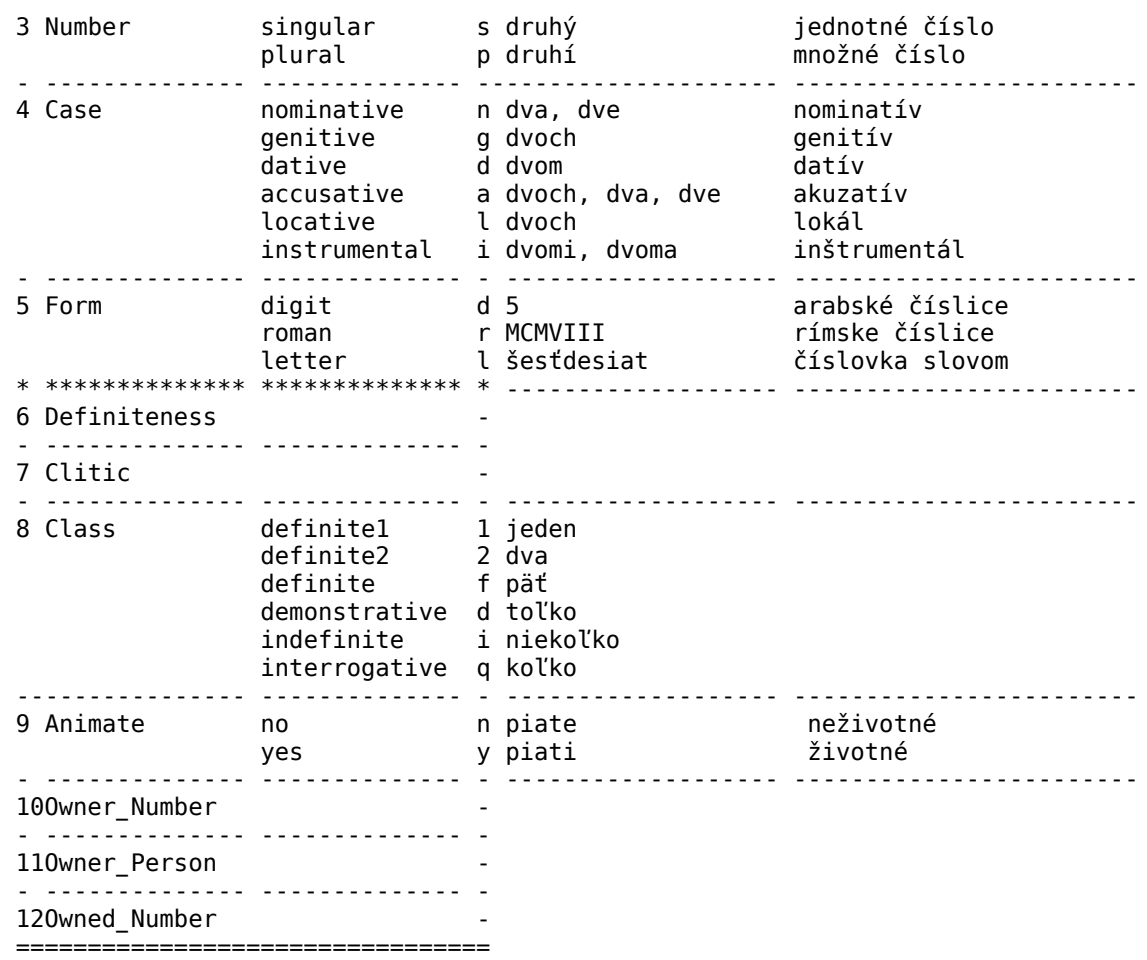

\section{Notes}

1. Numerals have been specified as a separate category because of their specific syntactic distribution. We have specified two syntactic classifications by means of the attributes Type and Class; they concern different syntactic distributions. For instance niekol"ko (E. "several") will be characterized as: Type: cardinal, Class: indefinite. Note that difference between pronouns and these classes of numerals is fuzzy and many words traditionally considered to be numerals are indeed classified as pronouns.

2. Among the definite numbers there are three subclasses (definite1, definite234, definite) which differ in their syntactic distribution and contain the following numerals: $\{1\},\{2,3,4\},\{5,6, \ldots\}$

3. Gender, Number and Case correspond to the same categories as specified for nouns. They are necessary for the proper account of agreement of numerals with nouns.

4. Cardinal numbers have the category Number assigned according to the cardinality of the noun they bind with. Notable exception is nula (zero), which binds with the plural, but has been assigned Number $=\mathrm{s}$ (ingular), because of the conflagration with homonymous noun-like form, which can be declined. 


\subsection{Combinations}

\begin{tabular}{|c|c|c|c|c|c|c|c|c|}
\hline$* * *$ & $* * * *$ & $* * * *$ & $* * * *$ & $* * * *$ & - - - & $-\cdots$ & $5-7$ & 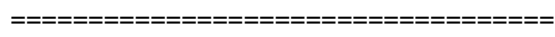 \\
\hline PoS & Type & Gend & Numb & Case & Form & Class & Anim & Examples \\
\hline$* * *$ & $* * * *$ & $* * * *$ & $* * * *$ & $* * * *$ & $-\ldots$ & $\ldots \ldots$ & $\ldots$ & 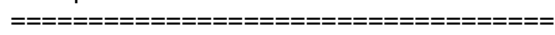 \\
\hline M & - & - & - & - & d & [123f] & - & 56 \\
\hline M & - & - & - & - & $r$ & [123f] & - & MVIII \\
\hline M & C & {$[\mathrm{mfn}]$} & $\mathrm{s}$ & any & l & 1 & - & jeden, jedna, jedno, nula \\
\hline M & C & {$[\mathrm{mfn}]$} & $\mathrm{p}$ & any & l & 2 & - & dva, dve \\
\hline M & C & - & $\mathrm{p}$ & any & l & 3 & - & tri, štyri \\
\hline M & C & $\mathrm{n}$ & [sp ] & any & l & $f$ & - & pät', desat', pät'desiat \\
\hline M & C & $\mathrm{n}$ & [sp] & any & l & [diq] & - & tol'ko, niekol'ko, kol'ko \\
\hline M & 0 & {$[\mathrm{mfn}]$} & [spd] & any & l & any & [ny- ] & prvý, druhý \\
\hline M & $\mathrm{m}$ & - & - & - & l & any & - & dvakrát, pät'krát, niekol’kokrát \\
\hline M & $\mathrm{s}$ & [mfn] & [spd] & $\mathrm{n}$ & l & any & {$[$ ny - ] } & dvoje, troje, pätoro \\
\hline *** & $* * * *$ & $* * * *$ & $* * *$ & & & & & \\
\hline
\end{tabular}

\section{Note}

In the Combinations above, 'any' is a variable standing for any admissible value.

\section{Interjection (I)}

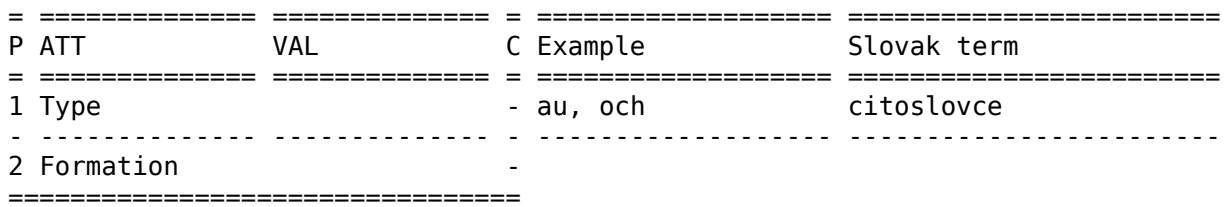

\section{Residual (X)}

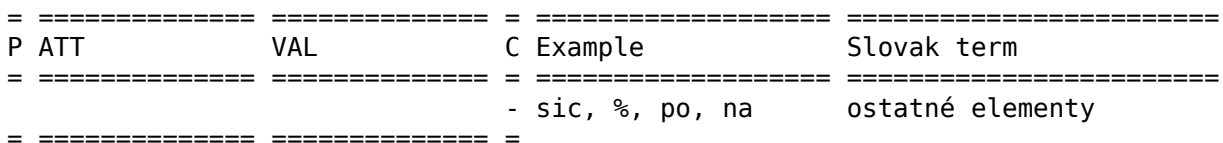

\section{Note}

Special "adverb prepositions" po, $n a$, do, encountered in expressions like $p o$ anglicky, na zeleno, do modra are classified as residuals. Traditional Slovak grammars do not like to consider them separate words, but more like modifiers of a following adverb. 


\section{Abbreviation (Y)}

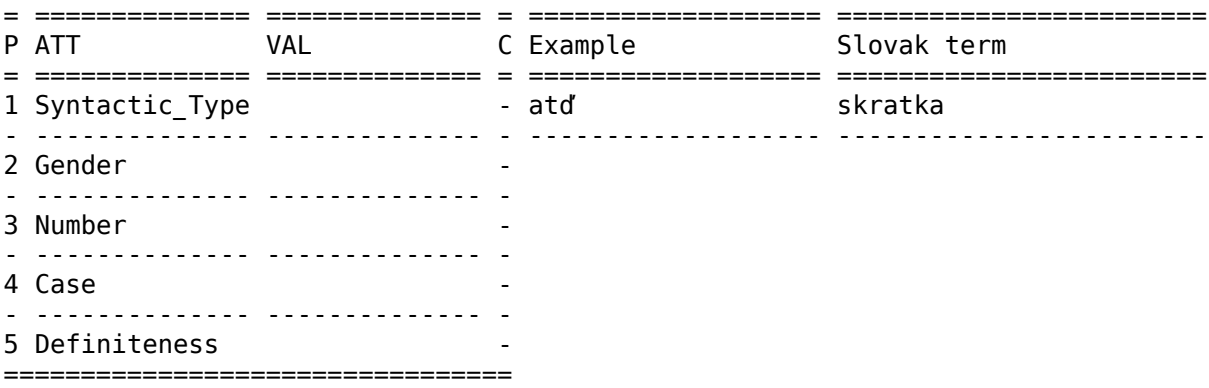

\section{Note}

As a result of our tokenizer, abbreviations do not contain the following full stop (the full stop is analysed as a punctuation character).

\section{Particle (Q)}

\begin{tabular}{|c|c|c|c|}
\hline$======= \pm===$ & $===========$ & $=0=0=0=0=0=0=0=0$ & 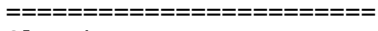 \\
\hline ATT & VAL & C Example & Slovak term \\
\hline$======ニ==ニ==$ & 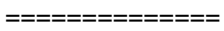 & $================$ & 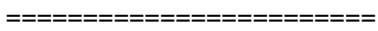 \\
\hline Type & & - áno, nie & častica \\
\hline---------- & ---------- & 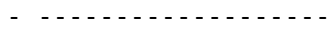 & 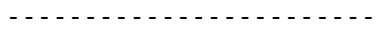 \\
\hline Formation & & - & \\
\hline------------ & ----------- & - & \\
\hline Clitic & & - & \\
\hline
\end{tabular}

\section{Note}

Traditionally, Slavic languages grammars recognise a separate word class of particles. The particle classification can be very detailed and complicated, reflecting their syntactical and semantic roles (Šimková, 2004). For the sake of clarity, we refrained from any classification and do not introduce any particle categories.

\section{Conclusion}

Designing a usable morphosyntactic tagsed is a non-trivial task, and there are many ways how to analyse the grammar categories in a way suitable for formal category assignment. Indeed, often there are several competing morphosyntactic tagsets for a given language, differing not at their surface forms, but deep in the underlying analysis and mapping of grammar features. Tagsets across different languages differ even more, which complicates cross linguistic research or analysis. Given that most 
of the Slavic language have their MTE specifications, MTE is probably the closest thing to a unified tagset for Slavic languages. Presented specification adds the Slovak language to the increasing set of languages that can benefit form existing MULTEXT-East tagset. The specification follows Multext-East V3; Slovak language specification has been officially included in MULTEXT-East V4, but from a linguistic point of view, the specifications are the same in V3 and V4; changes in V4 concern mostly internal data format (which is now in XML).

\section{References}

DIMITROVA, Ludmila - ERJAVEC, Tomaz - IDE, Nancy - KAALEP, Heiki Jaan - PETKEVIČ, Vladimir - TUFIŞ, Dan: Multext-East: Parallel and Comparable Corpora and Lexicons for Six Central and Eastern European Languages. In: Proceedings of COLING-ACL'98. Montréal - Québec 1998. pp. $315-319$.

GARABÍK, Radovan - GIANITSOVÁ, Lucia - HORÁK, Alexander - ŠIMKOVÁ, Mária ŠMOTLÁK, Martin: Slovak National Corpus. In: Proceedings of the conference TSD 2004. Brno: Springer-Verlag 2004.

GARABÍK, Radovan: Slovak Morphology Analyzer Based on Levenshtein Edit Operations. In: Proceedings of the WIKT'06 conference. Bratislava: 2006. pp. 2 - 5.

HAJIČ, Jan - VIDOVÁ-HLADKÁ, Barbora: Morfologické značkování korpusu českých textů stochastickou metodou. In: Slovo a slovesnost, 1997, roč. 58, č. 4, pp. 288 - 304.

IDE, Nancy - VÉRONIS, Jean: Multext (multilingual tools and corpora). In COLING'94, Kyoto 1994. pp. $90-96$.

Morfológia slovenského jazyka. Ed. J. Ružička. Bratislava: Vydavatel'stvo Slovenskej akadémie vied 1966.

MTE 2004: MULTEXT-East Morphosyntactic Specifications - version 3, edition 10 ${ }^{\text {th }}$. May 2004.

SEDLÁČEK, Radek - SMRŽ, Pavel: A New Czech Morphological Analyser AJKA. In: Proceedings of TSD. Berlin: Springer Verlag 2001. pp.100 - 107.

ŠIMKOVÁ, Mária: Funkcie častíc v komunikácii. In: Jazyk v komunikácii. Medzinárodný zborník venovaný Jánovi Bosákovi. Ed. S. Mislovičová, Bratislava: Veda 2004. pp. 168 - 176.

\section{Rés u mé}

V rámci projektu EC MULTEXT Multilingual Tools and Corpora vznikli vol'ne prístupné lingvistické zdroje a nástroje pre viaceré západoeurópske jazyky: angličtinu, francúzštinu, španielčinu, taliančinu, nemčinu, holandčinu a švédčinu. EC INCO-Copernicus projekt MULTEXT-East Multilingual Text Tools and Corpora for Central and Eastern European Languages nadviazal na predchádzajúci projekt a použitím rovnakej metodológie vyvinul podobné zdroje pre šest' d'alších jazykov: bulharčinu, češtinu, estónčinu, mad'arčinu, rumunčinu, slovinčinu a angličtinu. Slovenský jazyk netvoril súčast' projektu MULTEXT-East; tagset popísaný v článku vznikol nezávisle na pôde Jazykovedného ústavu L'. Štúra SAV v Bratislave s ciel’om dosiahnut' kompatibilitu s existujúcou špecifikáciou. Článok popisuje verziu tagsetu č. 3; v súčasnosti existuje novšia verzia č. 4, ktorá sa líši iba vo formáte uložených dát, z lingvistického pohl'adu nie je medzi verziou 3 a 4 podstatný rozdiel. 
Formát článku úmyselne dodržuje konvencie zaužívané v dokumentácii projektu MULTEXT-East, obzvlášt' vo formátovaní tabuliek a poznámok. Predpokladáme, že čitatel' je oboznámený s projektom MULTEXT-East alebo s MULTEXT-East tagsetom nejakého iného jazyka. Predkladaný tagset bol pragmaticky ovplyvnený morfosyntaktickým tagsetom slovenského jazyka používanom pri značkovaní Slovenského národného korpusu - dokonca bol úspešne vypracovaný automatický konvertor tagsetu Slovenského národného korpusu do tagsetu MULTEXT-East.

Navrhnutie použitel'ného, vnútorne konzistentného morfosyntaktického tagsetu je pomerne náročná úloha, aj s ohl'adom na rôzne možnosti analýzy gramatických javov v jazyku. Takáto úloha je ovel'a t'ažšia, ak berieme do úvahy aj iné jazyky s ciel'om zachytit' v tagsetoch ich morfologickú podobnost' (alebo odlišnost'). Ked'že pre takmer všetky slovanské jazyky existuje MULTEXT-East špecifikácia, používaný MULTEXT-East tagset má pravdepodobne najbližšie k (neexistujúcemu) univerzálnemu tagsetu slovanských jazykov. 